\title{
MACHT GOOGLE AUTONOM? \\ Zur Ambivalenz informationeller Autonomie
}

\author{
RAINER KUHLEN
}

Jeder weiß, was Google und andere Anbieter von Metainformationen leisten. Keine Frage, dass sie die Möglichkeit zum informationell autonomen Handeln erhöhen. Jeder denkt dabei zuerst an die Suchmaschine Google. Was immer auch deren unzählige Bewertungen im Detail besagen - einig sind sich in der Praxis alle: Man benutzt Google. Und die Konsequenz ist auch klar: Mit den Websuchdiensten, allen voran Google, hat sich die Art, wie wir auf Informationen zugreifen, und unsere Einstellung zu Wissen und Information grundlegend verändert.

Aber Suchmaschinen sind inzwischen nicht mehr nur zum Suchen da: Google und seine Konkurrenten erweitern ihr Angebot, indem sie die Produkte anderer übernehmen und weiterentwickeln. Ein Beispiel ist die Bildersuch- und -verwaltungssoftware von Picasa [@1]. Der Vorteil für den Verbraucher ist dabei, dass die Software jetzt kostenlos nutzbar und in den Google-Blogger-Service und natürlich den Google-Mail-Dienst Gmail integrierbar ist. Nutzen werden viele auch die Google-DesktopSoftware, mit der die Dateien auf dem eigenen Rechner komfortabel durchsucht werden können - bevorzugt solche, die mit Microsoft-Software erstellt wurden. Und so wird es weiter gehen: Googles Ziel »to organize the world's information, making it universally accessible and useful« [@2] ist fest im Blick.

Zukünftig wird Googles Angebot eine virtuelle Bibliothek einschließen, in der die gesamten Bestände großer Hochschulbibliotheken wie Stanford, Harvard oder Oxford elektronisch aufbereitet zugänglich sind zwei Jahrzehnte wird es nach Schätzung von Google dauern, bis dieses Projekt abgeschlossen ist. Der Einstieg in das Publikationsgeschäft wurde jüngst mit CrossRef Search gemacht [@3]: Google bereitet die Daten eines kommerziellen Dokumentlieferdienst auf und bietet die Ergebnisse auf der Website der beteiligten Verlage im Google-Format zur Suche an.

Das alles erinnert an Erfolgsgeschichten weit zurückliegender Informationsleistungen. Erinnern wir uns an das große Projekt der Aufklärung, dessen Sprengkraft nicht zuletzt darauf beruhte, dass die Bürgerinnen und Bürger von den Wissensvorsprüngen des Adels und Klerus 
unabhängig wurden: Gemeint ist die Enzyklopädie von Jean le Rond d' Alembert, Denis Diderot, Charles-Louis de Secondat de Montesquieu, Jean-Jacques Rousseau, Voltaire und vielen anderen Intellektuellen. Sie war bei ihrem Abschluss 1772 mit 25.000 verkauften Exemplaren das erfolgreichste Buchprojekt des 18. Jahrhunderts.

Mit Wissen war für diese Enzyklopädie nicht nur das "Hochwissen « gemeint, sondern auch das Alltagswissen, mit dem uns Google heutzutage versorgt. Das Rezept zum Anfertigen von Trinkschokolade, das durch Diderots Artikel in der Enzyklopädie weltberühmt wurde, kann selbstverständlich auch über Google gefunden und mit den ähnlichen Rezepten verglichen werden:

4 Esslöffel Schokolade, 2 Esslöffel Puderzucker, 3 Prisen Zimt, 1 Ei, Wasser \& Milch hinzufügen, im Wasserbad schmelzen; zum Schluss einen Tropfen Orangenblütenwasser und zwei Tropfen Ambra-Essenz hinzufügen.

Dies ist noch keine informationelle Autonomie, aber auch keine informationelle Abhängigkeit oder Fremdbestimmung - die informationelle Kapazität jedes Einzelnen kann dank Google stetig zunehmen. Nicht zuletzt der Konkurrenzdruck von Yahoo! und MSN Search wird dafür sorgen.

\section{Informationelle Autonomie}

Autonomie gehört zu den zentralen Konzepten europäischer Kultur. Selbstbestimmt handeln zu können, das ist die Forderung der europäischen Aufklärung und ein bis in die Gegenwart einzulösendes Projekt. Auf den Punkte brachte Immanuel Kant den Begriff Autonomie: Menschen sind autonom bzw. können als frei gelten, wenn sie sich in ihrem sittlichen Handeln nicht durch materielle oder sonstige Interessen bestimmen lassen, sondern wenn ihr Wille durch das von der Vernunft bestimmte allgemeine Sittengesetz geleitet wird. Dazu kann Google wenig beitragen.

Eine praktisch-politische Dimension sieht selbstbestimmtes, also autonomes Handeln in Abhängigkeit von der Verfügung über Wissen und Information. Selbstbestimmtheit kann sehr eng mit dem Begriff der Privatheit zusammenhängen. Dies stellt jedenfalls Beate Rössler (2003: 29) fest, wenn sie die Einlösung des Autonomieanspruchs eines jeden Individuums auch davon abhängig macht, inwieweit der Einzelne die Kontrolle darüber hat, was andere über ihn wissen und in welchem Umfang und unter welchen Bedingungen sie dieses Wissen verwenden dürfen. 
Entscheidend für Autonomie ist dies nach Rössler deshalb, weil man sich nur dann gegenüber anderen selbstbestimmt verhalten kann, wenn man weiß, was diese über einen selber wissen (vgl. Kuhlen 2004: 189). Eingang gefunden hat dieses Konzept der informationellen Privatheit in das so genannte Volkszählungsurteil des Bundesverfassungsgerichts von 1983, das sich damals primär gegen übertriebene Datenansprüche des Staates richtete und das über den Begriff der informationellen Selbstbestimmung jedem Bürger das Recht zubilligte, über die Erhebung, Verarbeitung und Weitergabe der ihn betreffenden Daten grundsätzlich selbst entscheiden zu dürfen.

\section{Google und Autonomie}

Noch sind wir nicht beim Thema, ob Google autonom macht, sondern vielmehr bei der Frage "Lässt uns Google autonom?«. Diese ist nicht zuletzt im Zusammenhang mit der Einführung von Gmail in der Netzöffentlichkeit breit diskutiert worden. Google selbst weist am 14. Januar 2005 ungefähr 1.280.000 Treffer für gmail privacy nach; der Konkurrent Yahoo! immerhin noch ca. 710.000 Einträge.

Google hat eine Fremdnutzung der Daten, die bei Gmail durch EMails anfallen und die auf individuelle Personen referenzierbar sind, wie bei den Suchanfragen, strikt ausgeschlossen - auch wenn es in der Google Policy [@4] viele Ausnahmen gibt. Im Sinne der Rössler'schen Bestimmung von informationeller Autonomie ist es problematisch genug, dass Google selbst so viel über uns weiß. Dies ist genug, um kontextbezogene Werbung anbieten zu können, nicht nur bei sich, sondern auch über Bereitstellung des dadurch entstandenen Know-hows bei anderen.

So gut wie jeder Internet-Anbieter hat solches Wissen über seine Nutzer; manches trägt zum Service bei. Ob die kontextstimmige Werbung als Infomercial dazu gehört, wie Google es annimmt, ist sicherlich strittig. Positiver wird vermutlich der ebenfalls aus dem Nutzungsverhalten entstandene Service des kollaborativen Filterns empfunden, der etwa Amazon in die Lage versetzt, passende Zusatzangebote zu einer aktuellen Buchbestellung bereitzustellen. Ein Unbehagen jedoch bleibt: Entscheiden wir noch autonom, wenn wir leicht zwanghaft dem folgen, was schon viele andere ebenfalls für gut befunden haben? Zumal kaum jemand weiß, worauf diese Zusatzinformationen wirklich beruhen.

\section{Das Vertrauen in Maschinen}

Der grundsätzliche Manipulations- und Missbrauchsverdacht ist im Netz immer vorhanden, egal ob es ein anfängliches Misstrauen oder ein durch Erfahrung verstärkter Verdacht ist. Sind die Daten erst einmal erhoben, könnten sie, so vermuten wir, wie der einmal aus der Flasche entwichene 
Geist nicht mehr kontrolliert werden. Die Versuchung, die Daten zu nutzen, ist einfach zu groß. Allerdings scheint der Verdacht nur wenige wirklich zu bekümmern. Das Vertrauen, besser die Vertrauensseligkeit oder Gleichgültigkeit, im Netz ist bei der Mehrheit grenzenlos. Die Ergebnisse der Suchmaschinen, um nur dabei zu bleiben, werden wie Naturgesetze akzeptiert. Was oben in den Ergebnislisten auftaucht, steht kaum in Zweifel gezogen - dort zu Recht.

Für die von Google und anderen gelieferten Metainformationen triff dabei das zu, was Psychologen wie Nass und Reeves (Nass et.al. 1995) oder Fogg (2003) empirisch belegt haben und was schon vor über 25 Jahren Weizenbaum mit seinen Eliza-Experimenten herausgefunden hatte: Wir räumen Software-Angeboten systematisch einen Kredit ein, der intelligenten Leistungen vorbehalten schien, und wir gehen bereitwillig auf deren Angebote ein, wenn sie denn nur brauchbar sind (Usability) und zum allgemeinen Spaß (Fun) oder Wohlbefinden (Joy) beitragen.

Warum ist das so? Wir delegieren demnach bereitwillig unsere Informationskompetenz an Programme, oder anders gesagt: Wir stecken Vertrauen in Leistungen, wo höchstes Misstrauen angebracht wäre. Etwa dann, wenn wir die Zuständigkeit für die informationelle Absicherung unseres Handelns abgeben an die vielfältigen externen Informationsressourcen, vor allem an die Informationsmaschinen. Gewinnen oder verlieren wir dabei?

Anthony Giddens (1990) geht von der These aus, dass Vertrauen dann zwangsläufig nötig ist und gegeben wird, wenn wir uns komplexen Systemen gegenübersehen, die wir prinzipiell nicht verstehen können. Vertrauen als Kompensation für systematisch bedingte Unsicherheit scheint fast schon perfide: Je mehr es den großen Systemanbietern, den Anbietern von Metainformation, gelingt, ihre Systemleistungen auf solch komplexe Verfahren zu stützen, dass sie kaum noch von den Experten durchschaut werden können, desto höher ist die Wahrscheinlichkeit, dass diesen Leistungen blind vertraut wird. Nicht ganz blind allerdings, denn irgendwelche Gründe haben wir in der Regel, wenn wir vertrauen: gute, d.h. hier nicht enttäuschte Erfahrung oder Referenzen anderer, denen wir als Experten zutrauen, dass sie den Durchblick haben - in der Regel also Übertragungsleistungen, kaum Einschätzungen auf Grund eigener Kompetenz bzw. Urteilskraft (vgl. Kuhlen 1999).

\section{Google als Metainformant}

Damit geraten wir in eine Situation, die zunächst gar nicht als problematisch erscheint. Zu Beginn des Beitrags haben wir autonomes Handeln aus einer praktisch-politischen Sicht betrachtet und dabei auf die Verfügung über Wissen und Information bezogen. Das war ja auch die Inten- 
tion der erwähnten Enzyklopädie als Aufklärungsprojekt. Anders als das Bundesverfassungsgericht bzw. ergänzend zur Perspektive des Datenschutzes haben wir winformationelle Selbstbestimmung " auf das Recht eines jeden Individuums bezogen, seine privaten, professionellen und öffentlichen Geschäfte informationell abgesichert abwickeln zu können. Gibt uns Google - vielleicht ohne zu wissen, auf welcher Grundlage diese Verfügung über Wissen und Information?

Vorderhand ja, denn Google leistet nichts anderes, als jedermann zumindest der Möglichkeit nach in die Lage zu versetzen, seine Geschäfte informationell abgesichert durchführen zu können. Das ist kein bloß potenzieller Dienst, wie ihn viele traditionelle Informations- und Dokumentationsstellen oder Bibliotheken leisten, sondern ein ganz realer täglich viele Millionen Mal in Anspruch genommen. Ein Tag ohne Google - ich verwende hier "Google«, so wie man Tempo für Papiertaschentücher oder Uhu für Alleskleber verwendet - ist für InternetNutzer kaum vorstellbar. Warum ist das so?

Sicher trifft es zu, dass wir einfach nicht mehr die Geduld haben, in eine Bibliothek zu gehen, in einem Buch so lange zu lesen oder auch nur zu blättern, bis wir die Stelle gefunden haben, die Auskunft gibt. Oder die Person anzurufen, von der wir vermuten, dass sie weiß, was wir wissen möchten. Warum aus Sentimentalität auf obsolet gewordene mediale Formen zurückgreifen oder sich auf ineffiziente Kommunikationssituationen einlassen, wenn das elektronische Mittel in einer allgegenwärtigen Online-Lebenswelt - am Arbeitsplatz und zu Hause sowieso, aber zunehmend auch unterwegs - immer zur Hand ist. Bücher, einschließlich Wörterbücher, Bibliotheken u.a., sind keine naturgegebenen Formen, um unsere menschlichen Defizite der Gedächtnis- und Informationsverarbeitung zu kompensieren; sie sind in Abhängigkeit von dem technisch-medialen Stand der jeweiligen Zeit entstanden. Und als solche bleiben sie uns auch noch eine Weile erhalten. Die Frage, ob die Bücher in ihrer traditionellen, zwischen zwei Einbände eingebundenen materiellen, Form durch elektronische Dienste verschwinden, ist für die Gegenwart und sicher auch für die überschaubare Zukunft beantwortet: natürlich nicht. Aber wohl schon die meisten Formen von Metainformation dürften hiervon betroffen sein. Und die liefern die Googles: Information und Information über Information (also Metainformation). Google liefert natürlich nicht Wissen, denn mit Wissen kann man schlecht Geld einnehmen. Google verdient Geld mit Information - direkt, indem die von Google erzeugte Metainformation an andere Informationsanbieter verkauft wird, indirekt, indem Dritte dafür bezahlen, dass Werbung für ihre thematisch zur Suchanfrage passenden Produkte und Dienstleistungen angezeigt wird. 


\section{Information und Wissen als Ware}

Typisch für die Informationsgesellschaft ist, dass wir offenbar mit der Information über Wissen mehr Geld machen und erst recht mehr Einfluss gewinnen können als mit den Wissensobjekten selbst. Die Sekundäroder Tertiärverwerter machen das Geschäft. Allerdings scheint das Geschäft nicht direkt zu Lasten der Verbraucher gehen zu müssen. Sie profitieren ja von dem Geschäftsmodell der Metainformationsanbieter. Bei den Suchmaschinen ist die Information über die Information dieser Wissensobjekte in der Regel gebührenfrei (wenn auch nicht unbedingt bedingungsfrei). Das könnte zum Paradigma in elektronischen Räumen werden: Die Information selbst ist frei (auch im Sinne von kostenfrei), verdient wird mit den Nebeneffekten (die Anbieter nennen das Mehrwertleistungen), die als eigentlicher kommerzieller Hauptzweck der Dienste kaum zu durchschauen sind.

Noch ist diese Kostenfreiheit ein Problem für kommerzielle Informationsanbieter wie Verlage, die nach wie vor mit ihren immateriellen Gütern, wie den Artikeln in den elektronischen Zeitschriften, verdienen wollen. Aber auch hier ist natürlich die Metainformation darüber frei, wie man zu den Informationen über die dann kostenpflichtigen Wissensobjekte kommen kann. Das Verlagskonsortium ScienceDirect [@5], um nur den derzeit wohl größten kommerziellen Dokumentnachweis- und -auslieferungsdienst zu nennen, bietet entsprechend zunächst nichts anderes als den Service einer Suchmaschine an. ScienceDirect (ähnlich die Elsevier-Suchmaschine Scirus [@6]) gibt Auskunft, welche Publikationen von welchem Verlag elektronisch verfügbar sind. Zur Relevanz- und damit zur Kaufentscheidung (die in Wirklichkeit keinen Kauf provoziert, sondern den Erwerb einer Nutzungslizenz) wird zusätzlich gebührenfrei das jeweilige Abstract, zuweilen weitere Information wie zitierte Literatur angeboten. Teuer wird es dann, wenn die das Wissen repräsentierenden Objekte, also die Aufsätze, angefordert werden.

\section{Informationskompetenz vs. Bildungsautonomie}

Die Googles sind auch deshalb so erfolgreich, weil wir heute viel mehr Informationsbedürfnisse haben als früher und weil wir keine Geduld mehr haben, auf die Antworten zu warten. Wir glauben immer häufiger von Dingen und Vorgängen, über die wir nicht ausreichend Bescheid wissen, mehr wissen $z u$ müssen - häufig auch zu Recht. Die Antworten kommen dabei schneller, als es die meisten Computerspezialisten noch vor wenigen Jahren für möglich gehalten hätten: Ohne Zeitverzögerung können viele Millionen Menschen gleichzeitig in Milliarden Informationsbeständen fündig werden. 
Dabei finden wir erneut Information, nicht Wissen. Das endlich führt uns zu dem entscheidenden Aspekt der informationellen Autonomie. Beruht die Erfolgsgeschichte der Suchmaschinen vielleicht auf etwas ganz anderem, nämlich darauf, dass sich das Verhältnis von Wissen und Information in den elektronischen Räumen radikal verändert hat? Gehen wir noch einmal auf die ursprünglichen Konzepte des autonomen Bürgers als Forderung und Programm der Aufklärung zurück. $\mathrm{Zu}$ Beginn des Aufsatzes "Beantwortung der Frage: Was ist Aufklärung?« (1784) von Immanuel Kant heißt es:

»Aufklärung ist der Ausgang des Menschen aus seiner selbstverschuldeten Unmündigkeit. Unmündigkeit ist das Unvermögen, sich seines Verstandes ohne Leitung eines anderen zu bedienen. Selbstverschuldet ist diese Unmündigkeit, wenn die Ursache derselben nicht am Mangel des Verstandes, sondern der Entschließung und des Mutes liegt, sich seiner ohne Leitung eines anderen zu bedienen. Sapere aude! Habe Mut, dich deines eigenen Verstandes zu bedienen! ist also der Wahlspruch der Aufklärung«.

Dem Zitat entnehmen wir einen anderen Kompetenzbegriff als den, den wir aus unserer Diskussion über die Metainformationsdienstleistungen gewonnen haben. Da haben wir von Informationskompetenz als der Fähigkeit gesprochen, sich der Ressourcen bedienen zu können, die es erlauben, Geschäfte (welcher Art auch immer), informationell abgesichert durchführen zu können. Kant sah das noch skeptisch, auch, was das Vertrauen gegenüber Informationsdienstleistungen anderer anbetraf. In einer anderen Stelle des gleichen Aufsatzes heißt es:

"Habe ich ein Buch, das für mich Verstand hat, einen Seelsorger, der für mich Gewissen hat, einen Arzt, der für mich die Diät beurteilt, usw.: so brauche ich mich ja nicht selbst zu bemühen. Ich habe nicht nötig zu denken, wenn ich nur bezahlen kann; andere werden das verdrießliche Geschäft schon für mich übernehmen «.

Kant spricht von Wissensautonomie, vielleicht aber auch nur von der Fähigkeit, über ausreichende Urteilskraft zu verfügen, um die angetragene bzw. aufgefundene Information in ihrem Wahrheitswert und ihrer Handlungsrelevanz einschätzen zu können. Entsprechend dieses Bildungskonzepts bedeutete Autonomie lange Zeit immer auch schon Wissensautonomie, zumindest als regulatives Ziel, obwohl faktisch niemand je hat behaupten können, all das zu wissen, was in realen Situationen an Wissen gebraucht wird. Dieses Autonomieverständnis hat sich durch die gegenwärtigen medialen und technologischen Revolutionen grundlegend 
geändert. In Informationsgesellschaften wird Informationskompetenz und damit Informationsautonomie zum allgemeinen Bildungsziel. Sie ist die Freiheit und das Recht, auf bestehendes Wissen bzw. dessen Ressourcen zugreifen und die dafür erforderliche Kompetenz und Kapazität erwerben zu können. Trivialisieren wir das philosophische Konzept der Urteilskraft, dann sollte Informations- und Medienkompetenz zum zentralen Bildungsziel in der Google-Gesellschaft werden.

\section{Konsequenzen für die (Aus-)Bildung}

Allerdings - und das ist Grundlage für den Erfolg der Googles und vermutlich in der Zukunft weiterer intelligenterer Suchagenten - kann in vielen Fällen Informationsautonomie nicht mehr durchgängig selber wahrgenommen werden, zumal Informations- und Medienkompetenz bislang kaum real in den Ausbildungsgängen und Schulen vermittelt wird. Immer mehr wird Wissens- und Informationsarbeit ausgelagert. Andere (ob Menschen, Systeme oder intelligente maschinelle Assistenten) verrichten Informationsarbeit für uns, so wie traditionell andere Maschinen z.B. Mobilitätsarbeit für uns verrichten. Aber Auslagerung von Informationsarbeit, die ja den Zugriff zu Wissen ermöglichen soll, ist etwas anders, geht weiter. Informationsmaschinen übernehmen das, was bislang als Privileg von Menschen beim Umgang mit Wissen und Information angesehen wurde: gezieltes Suchen und Wiederfinden, Ableiten von Wissensstrukturen durch Vergleich, Planung und Durchführung von komplexen Handlungen auf der Grundlage des maschineninternen Wissens. Galten solche Fähigkeiten bislang als Merkmale menschlichen autonomen Informationshandelns, so werden sie bei der maschinellen Übernahme zu Merkmalen autonomer Technik.

Die angesprochene Auslagerung von Wissens- und Informationsarbeit bekommt damit eine neue Qualität. An Wissen wird nicht mehr nur das ausgelagert, was das einzelne Gedächtnis nicht speichern kann, sondern der Prozess der Erarbeitung von Information aus vorhandenen externen Wissensquellen (als Basis der Aneignung von neuem Wissen) beginnt ebenfalls sich in Richtung einer Technikautonomie zu verselbstständigen. Die Informationsmaschinen beginnen das als Problem zu definieren, was zunächst harmlos nur als Antwort auf die von uns gestellte Frage gedacht war. Und mehr noch: Informationelle Autonomie wird zum Selbstzweck. Wir erarbeiten uns Information nicht mehr, um uns diese durch den Zugriff auf das zu Grunde liegende Wissen dauerhaft anzueignen. Warum sollen wir auch, wenn wir uns das aktuell Gefundene und vielleicht auch Verwendete jederzeit mit minimalem Aufwand wie- 
der beschaffen können? Steht uns eine informationelle WegwerfGesellschaft mit (oder ohne) Wiederaufbereitungsanspruch bevor?

Das ist Teil dessen, was Googlerisierung der Ausbildung genannt werden kann, aber auch Teil der Googlerisierung der Gesellschaft insgesamt sein wird. Ersteres bedeutet nicht nur, dass Studierende kaum mehr Bibliotheken benutzen, kaum mehr von ihren Professoren angehalten werden, in den eigentlichen elektronischen Ressourcen des Wissens, nämlich den Online-Datenbanken der Fachinformation, zu suchen. Es bedeutet auch, dass Studierende nur noch das für fachliche Realität halten, was sie über Google finden. Diese dramatisch nachlassende oder überhaupt nicht gebildete Informationskompetenz ist empirisch gut in der vom BMBF geförderten SteFi-Studie nachgewiesen (vgl. Klatt et al. 2001). Dieses spezielle Defizit wird Google wahrscheinlich selbst beseitigen, wenn, wie schon am Beispiel der Digitalisierung von Bibliotheksbeständen angedeutet, die Suchmaschine sich auch die Rechte sichert, welche die bislang für das Web »dunklen « Bestände der Fachinformation erschließen. Dann wird auch Fachinformation Google-Information sein.

Diese kritischen Anmerkungen sind kein Appell, wieder zum Ideal der Wissensautonomie zurückzukehren. Das wäre eine Illusion. Wir werden mit den Googles leben müssen, und wir leben ja auch gerne damit, weil sie uns eine Autonomie vorgaukeln, die es so nie gegeben hat. $\mathrm{Zu}$ wirklicher Autonomie kann das allerdings nur führen, wenn die Informationsangebote der Googles nicht nur als konsumierbare Ware angesehen und schon gar nicht für bare Münze gehalten werden, sondern als Objekte, die es zu beurteilen, mit bestehendem Wissen zu vergleichen und zielgerecht einzusetzen gilt. Validität und Handlungsrelevanz zu erkennen, geht nur mit Urteilskraft - da haben die Philosophen sicher recht. Urteilskraft kann aber nur auf der Grundlage erworbenen Wissens operieren. Entsprechend erschöpft sich Informations- und Medienkompetenz nicht in der technischen Bedienung der Systeme, sondern sie beruht auf der Fähigkeit, das oft verborgene Wissen aus der Flut der Informationen herauszufiltern, zu bewerten und sich anzueignen. Das ist das aufklärerische Autonomie-Potenzial der Googles - wenn es denn gelingt, Information wieder in den Prozess der individuellen oder kollaborativen Wissensaneignung und -produktion zurückzubinden. Dazu gehört auch, Information nicht zum Ziel an sich und damit nicht zum Objekt des Vergessens, des beliebig Erneuerbaren, werden zu lassen. Google mag in der Informationsgesellschaft eine notwendige Bedingung für Autonomie sein, aber keinesfalls eine hinreichende. 


\section{Literatur}

Fogg, B.J. (2003): Persuasive Technology. Using Computers to Change What We Think and Do. Morgan Kaufmann Publishers: Amsterdam.

Giddens, Anthony (1990): The Consequences of Modernity. Stanford University Press, Stanford, CA.

Klatt, R./Gavriilidis, K./Kleinsimlinghaus, K./Feldmann, M. u.a. (2001): "Nutzung elektronischer wissenschaftlicher Information in der Hochschulausbildung. Barrieren und Potenziale der innovativen Mediennutzung im Lernalltag der Hochschulen. Endbericht Dortmund «. [WWW-Dokument: www.stefi.de/download/bericht2.pdf- Zugriff: 02.02.2005].

Kuhlen, Rainer (1999): Die Konsequenzen von technischen Assistenten. Was bedeutet informationelle Autonomie oder wie kann Vertrauen in Assistenten für elektronische Mediendienste in offenen Informationsmärkten gesichert werden? Suhrkamp: Frankfurt/Main.

Kuhlen, Rainer (2004): Informationsethik - Formen des Umgangs mit Wissen und Information. Universitätsverlag Konstanz, Konstanz.

Nass, C./Moon, Y./Fogg, B.J./Reeves, B. und D.C. Dryer: "Can Computer Personalities be Human Personalities? « In: International Journal HumanComputer Studies, 43, 23-239.

\section{Digitale Verweise}

[@1] www.picasa.com

[@2] www.google.com/contact/search.html

[@3] www.crossref.org/crossrefsearch.html

[@4] http://gmail.google.com/gmail/help/privacy.html\#disclose

[@5] www.sciencedirect.com

[@6] www.scirus.com/srsapp 